\title{
PcMab-47: Novel Antihuman Podocalyxin Monoclonal Antibody for Immunohistochemistry
}

\author{
Satoshi Ogasawara, ${ }^{1, *}$ Mika K. Kaneko,,2," Shinji Yamada,,2 Ryusuke Honma, Takuro Nakamura,,2 \\ Noriko Saidoh,,2 Miyuki Yanaka,, Kanae Yoshida,, Yuki Fujii, and Yukinari Kato ${ }^{1-3}$
}

Podocalyxin (PODXL) is a CD34-related sialomucin and a well-known marker of embryonic stem cells. PODXL is expressed in many types of tumors including colorectal cancers, breast cancers, and brain tumors. Overexpression of PODXL is an independent predictor of progression, metastasis, and poor outcome. PODXL is also expressed in many normal cells such as renal podocytes and endothelial cells (ECs). However, highsensitive and high-specific anti-PODXL monoclonal antibodies (mAbs) have not been established. Herein, we immunized mice with recombinant human PODXL, which was produced using LN229 glioblastoma cells. The anti-PODXL mAb, PcMab-47, reacted with endogenous PODXL-expressing cancer cell lines and normal cells independently of glycosylation in flow cytometry. Immunohistochemical analysis showed that PcMab-47 detected PODXL-expressing normal cells such as podocytes of kidney or ECs. Furthermore, PcMab-47 stained PODXL-expressing cancer cells of colon or breast cancers. These results suggest that PcMab-47 could be useful for investigating the expression and function of PODXL in cancers and normal tissues.

Keywords: podocalyxin, PODXL, monoclonal antibody, immunohistochemistry

\section{Introduction}

$\mathbf{P}$ ODOCALYXIN (PODXL), which is also known as PCLP, MEP21, Gp200, Gp135, thrombomucin, GCTM2, TRA1-60 antigen, or TRA-1-81 antigen, is a type I transmembrane protein with a molecular weight of $150,000-200,000 .^{(1-3)}$ PODXL is expressed in normal tissues, including kidney, heart, pancreas, and breast tissues, as well as in neurons, ductal luminal cells, podocytes, endothelial cells (ECs), oviductal luminal cells, and mesothelial cells; PODXL also plays an important role in the development of tissues. ${ }^{(4)}$ PODXL is similar to CD34, which is known as a hematopoietic stem cell marker. ${ }^{(5)}$ A sialomucin, PODXL is highly glycosylated with $\mathrm{N}$-glycan, $\mathrm{O}$-glycan, and keratan sulfate. ${ }^{(6)}$ PODXL was first found in rat podocytes of the kidney, ${ }^{(7)}$ and its homologues have been reported in humans. ${ }^{(8,9)}$

PODXL has different functions in various tissues. PODXL acts as an adhesive molecule to bind to platelets or vascular endothelial cells (VECs) ${ }^{(10,11)}$; in contrast, PODXL functions as an antiadhesive molecule through its negatively charged mucin domain, including sialic acid and keratan sulfate, for the formation and maintenance of the filtration slits between the podocyte processes in the kidney. ${ }^{(12)}$ In addition, PODXL regulates cell morphology by associating with actin cytoskeletal proteins, such as $\mathrm{Na}^{+} / \mathrm{H}^{+}$exchanger regulatory factor (NHERF)-1/2 and ezrin. ${ }^{(13,14)}$ PODXL is also known as one of the pluripotent stem cell markers. The antigen of the monoclonal antibodies (mAbs), TRA-1-60 and TRA-1-81, is keratan sulfate, which is attached to PODXL. ${ }^{(3)}$ The ligand of the recombinant N-terminal domain of the lectin BC2L-C from Burkholderia cenocepacia $(\mathrm{rBC} 2 \mathrm{LCN})$ is also reported as $O$ glycan of PODXL. ${ }^{(15)}$

PODXL is known as a diagnostic marker and prognostic indicator in several cancers, including brain tumors, $(6,16)$ prostate cancers, ${ }^{(17)}$ testicular tumors, ${ }^{(2)}$ renal cancers, ${ }^{(18)}$ oral cancers, ${ }^{(19)}$ thyroid cancers, ${ }^{(20)}$ bladder cancers, ${ }^{(21)}$ breast cancers, ${ }^{(22-24)}$ ovarian cancers, ${ }^{(25)}$ colorectal cancers, ${ }^{(26-29)}$ pancreatic cancers, ${ }^{(30,31)}$ and gastric cancers. ${ }^{(32)}$ The glycans on PODXL bind to P-/E-/L-selectin expressed on platelets, endothelium, and leukocytes, respectively. ${ }^{(33-35)}$

Departments of ${ }^{1}$ Regional Innovation and ${ }^{2}$ Antibody Drug Development, Tohoku University Graduate School of Medicine, Sendai, Japan.

${ }^{3}$ New Industry Creation Hatchery Center, Tohoku University, Sendai, Japan.

*Contributed equally to this work.

(C) Satoshi Ogasawara et al., 2017; Published by Mary Ann Liebert, Inc. This Open Access article is distributed under the terms of the Creative Commons License (http://creativecommons.org/licenses/by/4.0), which permits unrestricted use, distribution, and reproduction in any medium, provided the original work is properly credited. 
These interactions enhance the formation of platelet-tumorleukocyte aggregates and tumor cell arrest in the microvasculature. ${ }^{(36)}$ Therefore, the overexpression of PODXL in cancer is a potential target for antibody therapy.

In this study, we established the anti-PODXL mAb, PcMab47 , for use in flow cytometry and immunohistochemistry.

\section{Materials and Methods}

\section{Cell lines}

LN229, Caco-2, MDA-MB-468, HEK-293T, Chinese hamster ovary (CHO)-K1, glycan-deficient $\mathrm{CHO}$ cell lines (Lec1, Lec2, and Lec8), and P3U1 were obtained from the

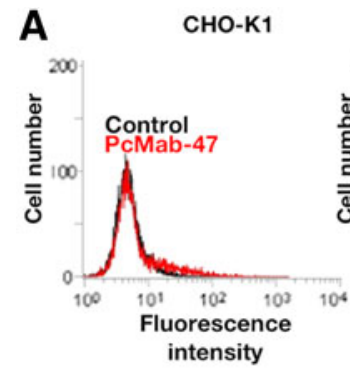

C

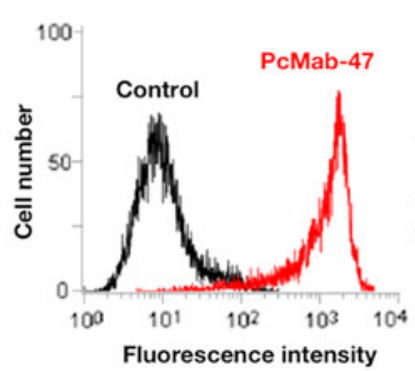

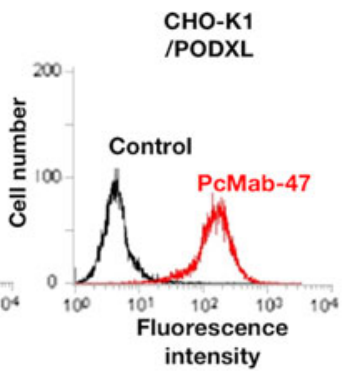

MDA-MB-468

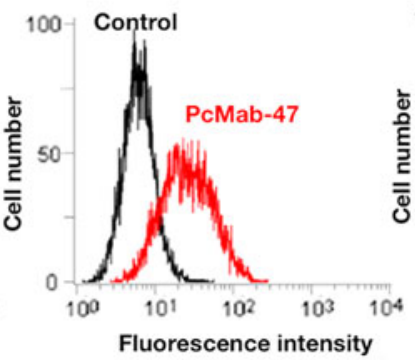

B

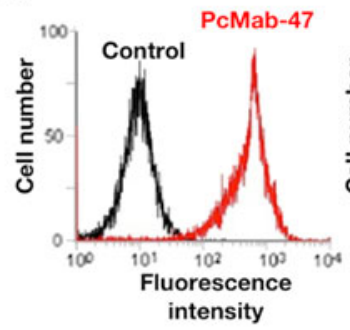

LN229/PODXL

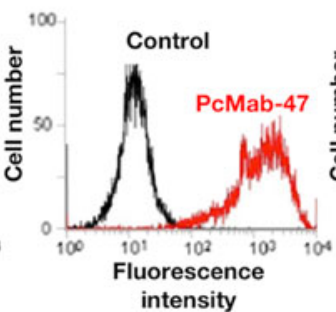

HEK-293T

PDIS-13

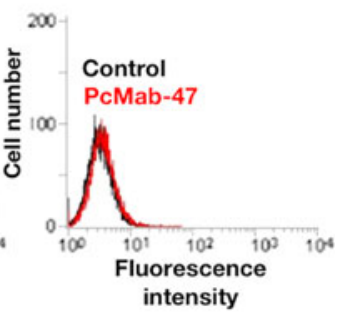

VEC
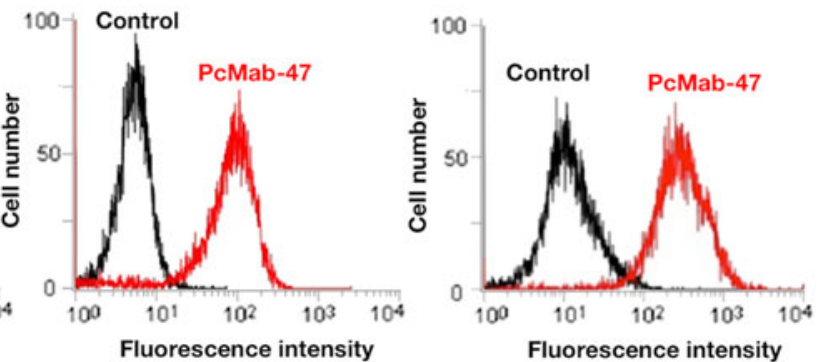

Lec8/PODXL
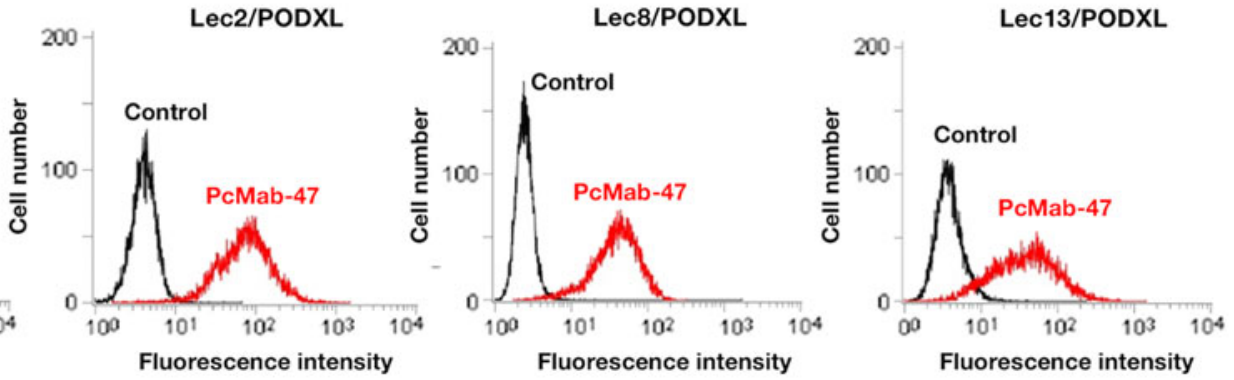

PDIS-22
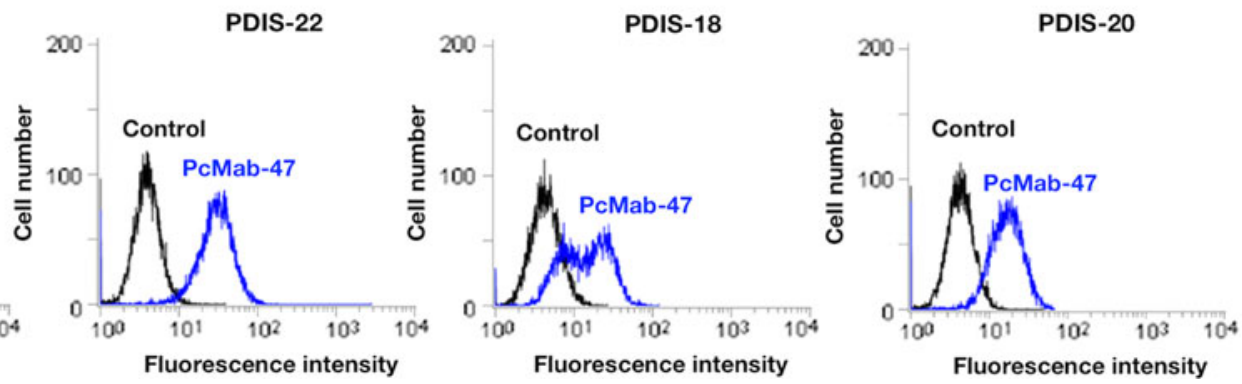

FIG. 1. Specific detection of PODXL by PcMab-47 using flow cytometry. (A) CHO-K1 and CHO-K1/PODXL were treated with PcMab-47 (1 $\mu \mathrm{g} / \mathrm{mL}$; red) or control PBS (black) for 30 minutes at $4{ }^{\circ} \mathrm{C}$, followed by treatment with antimouse IgG-Oregon green. (B) LN229, LN229/PODXL, and PDIS-13 (LN229/PODXL-knockout cells) were treated with PcMab-47 (1 $\mu \mathrm{g} / \mathrm{mL}$; red) or control PBS (black) for 30 minutes at $4{ }^{\circ} \mathrm{C}$, followed by treatment with antimouse IgG-Oregon green. (C) Caco-2, MDA-MB468, HEK-293T, and vascular endothelial cells were treated with PcMab-47 $(1 \mu \mathrm{g} / \mathrm{mL}$; red) or control PBS (black) for 30 minutes at $4^{\circ} \mathrm{C}$, followed by treatment with antimouse IgG-Oregon green. (D) Lec1 ( $N$-glycan-deficient CHO)/PODXL, Lec2 (sialic acid-deficient CHO)/PODXL, Lec8 (galactose-deficient CHO)/PODXL, and Lec13 (fucose-deficient CHO)/PODXL were treated with PcMab-47 ( $1 \mu \mathrm{g} / \mathrm{mL}$; red) or control PBS (black) for 30 minutes at $4{ }^{\circ} \mathrm{C}$, followed by treatment with antimouse IgG-Oregon green. (E) PDIS-12 ( $N$-glycan-deficient HEK-293T), PDIS-22 (sialic acid-deficient HEK-293T), PDIS-18 (galactose-deficient HEK-293T), and PDIS-20 ( $\mathrm{N}$-glycan/sialic acid/galactose-deficient HEK-293T) were treated with PcMab$47\left(1 \mu \mathrm{g} / \mathrm{mL}\right.$; blue) or control PBS (black) for 30 minutes at $4^{\circ} \mathrm{C}$, followed by treatment with antimouse IgG-Oregon green. Fluorescence data were collected using the Cell Analyzer EC800. PBS, phosphate buffered saline; PODXL, podocalyxin. 
American Type Culture Collection (Manassas, VA). Human VECs were purchased from Cambrex (Walkersville, MD). Lec13 was provided by Dr. Pamela Stanley.

LN229, Lec1, Lec2, Lec8, and Lec13 were transfected with PODXL plasmids, which included the ectodomain or full length of PODXL, using Lipofectamine 2000 (Thermo Fisher Scientific, Inc., Waltham, MA) according to the manufacturer's instructions. LN229/hPODXL-knockout (KO) cells (PDIS-13) were produced using CRISPR/Cas9 plasmids (Target ID: HS0000056763) against human PODXL (Sigma-Aldrich, St. Louis, MO).

The cell lines HEK-293T/GnT-1-KO (PDIS-12), HEK293T/SLC35A1-KO (PDIS-22), HEK-293T/SLC35A2-KO (PDIS-18), and HEK-293T/GnT-1/SLC35A1/SLC35A2-KO (PDIS-20) were generated by transfecting TALEN or CRISPR/Cas9 plasmids, which target hsMgat1 (Wako Pure Chemical Industries Ltd., Osaka, Japan), SLC35A1 (Target ID: HS0000168432; Sigma-Aldrich), and SLC35A2 (Target ID: HS0000062603; Sigma-Aldrich), respectively, using a Gene Pulser Xcell electroporation system, and were screened using lectin profiling. These glycan-deficient cell lines are available from Cell Bank of Kato's Lab (www.medtohoku-antibody.com/topics/001_paper_cell.htm) in Tohoku University (Miyagi, Japan).

CHO-K1, Lec1, Lec2, Lec8, Lec13, CHO-K1/PODXL, Lec1/PODXL, Lec2/PODXL, Lec8/PODXL, Lec13/PODXL, and P3U1 were cultured in RPMI 1640 medium, including Lglutamine (Nacalai Tesque, Inc., Kyoto, Japan). L-Proline $(0.04 \mathrm{mg} / \mathrm{mL})$ was added for Lec1, Lec2, Lec8, and Lec13. LN229, LN229/PODXL, LN229/ectodomain-PODXL, PDIS13, HEK-293T, Caco-2, MDA-MB-468, PDIS-12, PDIS-22, PDIS-18, and PDIS-20 were cultured in Dulbecco's modified Eagle's medium, including L-glutamine (Nacalai Tesque, Inc.), supplemented with $10 \%$ heat-inactivated fetal bovine serum (FBS; Thermo Fisher Scientific, Inc.) at $37^{\circ} \mathrm{C}$ in a humidified atmosphere of $5 \% \mathrm{CO}_{2}$ and $95 \%$ air. G418 $(0.5 \mathrm{mg} / \mathrm{mL}$; Wako Pure Chemical Industries Ltd.) was added for CHOK1/PODXL, Lec1/PODXL, Lec2/PODXL, Lec8/PODXL, Lec13/PODXL, LN229/PODXL, and LN229/ectodomainPODXL. VECs were cultured in EC medium EGM-2 MV supplemented with 5\% FBS (Cambrex Corp.). Antibiotics, including $100 \mathrm{U} / \mathrm{mL}$ of penicillin, $100 \mu \mathrm{g} / \mathrm{mL}$ of streptomycin, and $25 \mu \mathrm{g} / \mathrm{mL}$ of amphotericin B (Nacalai Tesque, Inc.), were added to all media.

\section{Hybridoma production}

Four-week-old female BALB/c mice (CLEA, Tokyo, Japan) were immunized by intraperitoneal (i.p.) injection of the purified ectodomain of human PODXL $(100 \mu \mathrm{g})$ together with Imject Alum (Thermo Fisher Scientific, Inc.). After several additional immunizations, a booster i.p. injection of LN229/PODXL was given 2 days before the mice were euthanized by cervical dislocation, and spleen cells were harvested. The spleen cells were fused with P3U1 cells using PEG1500 (Roche Diagnostics, Indianapolis, IN). Hybridomas were grown in RPMI 1640 medium including L-glutamine with hypoxanthine, aminopterin, and thymidine selection medium supplement (Thermo Fisher Scientific, Inc.). Culture supernatants were screened using enzyme-linked immunosorbent assay (ELISA) for binding to the purified ectodomain of PODXL. Proteins were immobilized on Nunc Maxisorp 96well immunoplates (Thermo Fisher Scientific, Inc.) at $1 \mu \mathrm{g} / \mathrm{mL}$ for 30 minutes. After blocking with $1 \%$ bovine serum albumin (BSA) in $0.05 \%$ Tween20/phosphate buffered saline (PBS; Nacalai Tesque, Inc.), the plates were incubated with culture supernatant followed by 1:2000 diluted peroxidase-conjugated antimouse IgG (Agilent Technologies, Inc., Santa Clara, CA).
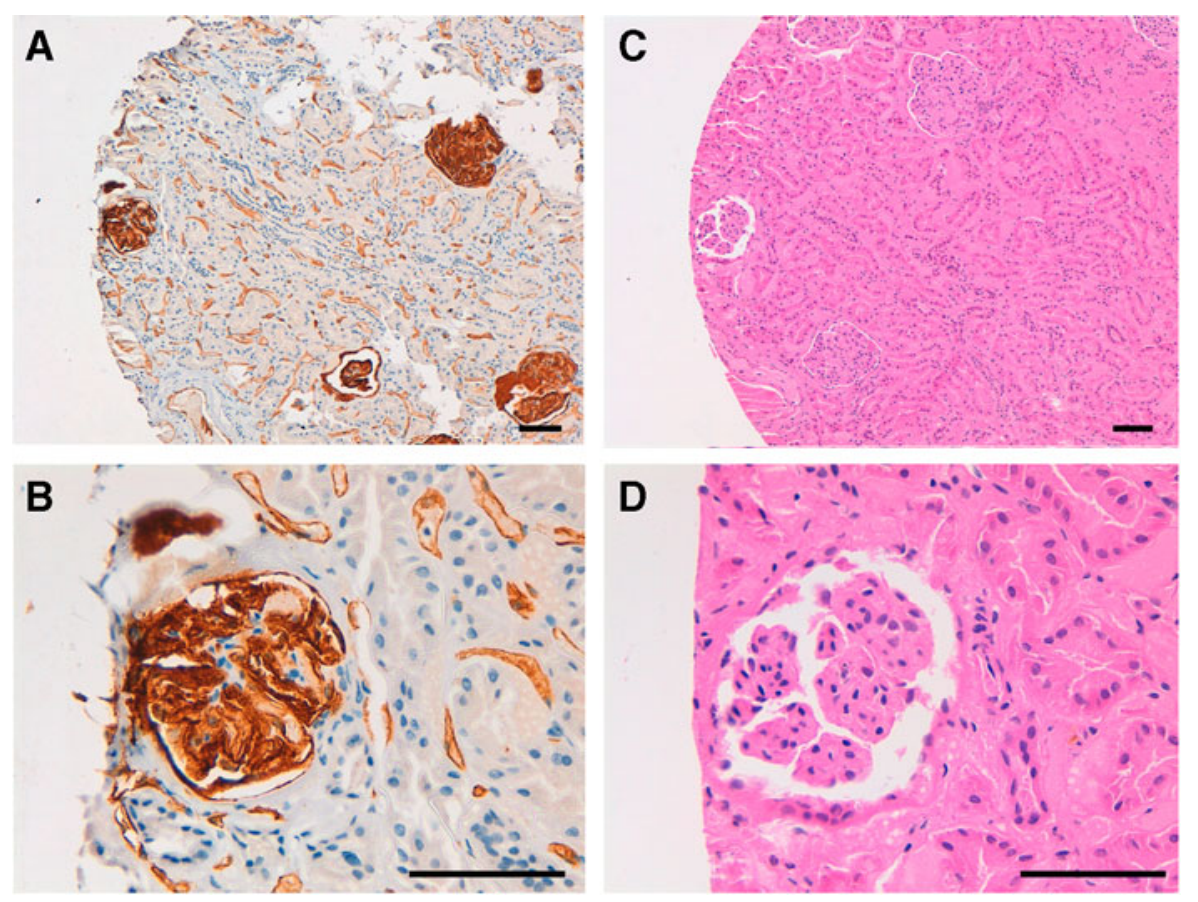

FIG. 2. Immunohistochemical analysis of human kidney by PcMab-47. A section of kidney was incubated with $1 \mu \mathrm{g} / \mathrm{mL}$ of PcMab-47, followed by the Envision+ kit (A, B). Color was developed using 3, 3-diaminobenzidine tetrahydrochloride, and counterstained with hematoxylin. Another section of kidney was also stained using hematoxylin and eosin (C, D). Scale bar: $100 \mu \mathrm{m}$. 
The enzymatic reaction was produced with a 1-Step Ultra TMB-ELISA (Thermo Fisher Scientific, Inc.). The optical density was measured at $655 \mathrm{~nm}$ using an iMark microplate reader (Bio-Rad Laboratories Inc., Hercules, CA).

\section{Flow cytometry}

Cell lines were harvested by brief exposure to $0.25 \%$ Trypsin/1 mM EDTA (Nacalai Tesque, Inc.). After washing with $0.1 \%$ BSA in PBS, cells were treated with primary mAbs
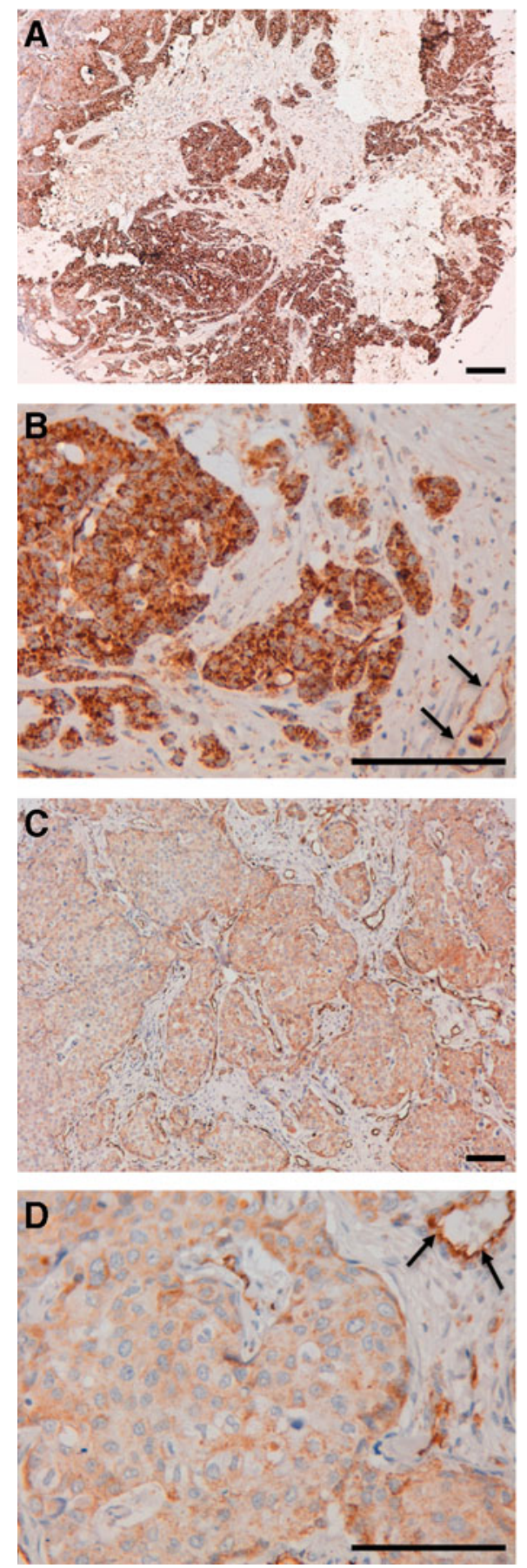

for 30 minutes at $4^{\circ} \mathrm{C}$, followed by treatment with Oregon Green 488 goat antimouse IgG (Thermo Fisher Scientific, Inc.). Fluorescence data were collected using the Cell Analyzer EC800 (Sony Corp., Tokyo, Japan).

\section{Immunohistochemical analyses}

Human normal tissues and cancer tissues were purchased from BioChain Institute, Inc. (Newark, CA) and US Biomax, Inc. (Rockville, MD), respectively. Four-micrometer-thick
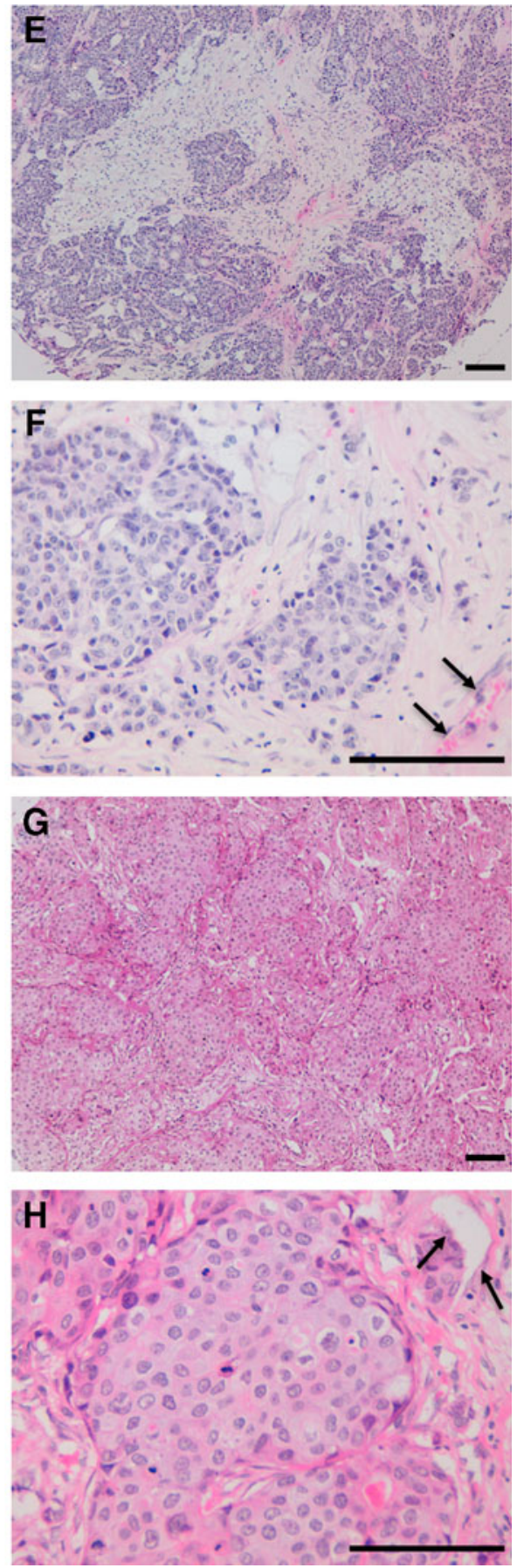

FIG. 3. Immunohistochemical analysis of human cancers by PcMab-47. Sections of colon cancer (A, B) and breast cancer (C, D) were incubated with $10 \mu \mathrm{g} / \mathrm{mL}$ of PcMab-47, followed by the Envision+ kit. Color was developed using 3, 3-diaminobenzidine tetrahydrochloride and counterstained with hematoxylin. Colon cancer $(\mathbf{E}, \mathbf{F})$ and breast cancer $(\mathbf{G}, \mathbf{H})$ were also stained using hematoxylin and eosin. Arrows: endothelial cells. Scale bar: $100 \mu \mathrm{m}$. 
histological sections were deparaffinized in xylene and rehydrated. After the antigen retrieval procedure (autoclave using citrate buffer, $\mathrm{pH}$ 6.0; Agilent Technologies, Inc.), sections were incubated with 1 or $10 \mu \mathrm{g} / \mathrm{mL}$ of PcMab- 47 for 1 or 3 hours at room temperature followed by treatment with Envision+ kit (Agilent Technologies, Inc.) for 30 minutes. Color was developed using 3, 3-diaminobenzidine tetrahydrochloride (Agilent Technologies, Inc.) for 5 minutes, and then the sections were counterstained with hematoxylin (Wako Pure Chemical Industries Ltd.).

\section{Results and Discussion}

In our previous study, we developed the original technology for production of cancer-specific monoclonal antibodies (CasMabs) against membranous proteins. ${ }^{(37)}$ We successfully produced the antipodoplanin (PDPN) CasMab clone LpMab-2 that specifically recognizes cancer-type PDPN in tumor tissues and not in normal-type PDPN, which is expressed in lymphatic vessels. ${ }^{(37)}$ Moreover, the CasMab technology is useful for generating antiglycopeptide mAbs (GpMabs). We have produced the following anti-PDPN GpMabs: LpMab-3, LpMab-9, LpMab-12, LpMab-19, and LpMab-21. ${ }^{(38-40)}$ In addition, the CasMab technology can generate mAbs that bind to various novel epitopes of PDPN. We have produced the following anti-PDPN mAbs: LpMab-7, LpMab-10, and LpMab-17, which recognize novel epitopes. ${ }^{(37,40-42)}$ This study describes the production of sensitive and specific anti-PODXL mAbs using the CasMab technology.

Herein, we immunized mice with recombinant PODXL, which was purified from the culture supernatant of LN229/ ectodomain-PODXL. A booster i.p. injection of LN229/ PODXL was administered. The culture supernatants were screened using ELISA for binding to purified PODXL. As a second screening, we performed flow cytometry for reaction with LN229 and LN229/PODXL. A stronger reaction against LN229/PODXL than against LN229 was necessary, and one clone, PcMab-47 (mouse $\mathrm{IgG}_{1}$, kappa), was produced after limiting dilution.

PcMab-47 reacted with CHO-K1/PODXL, and did not react with CHO (human PODXL-negative cell) in flow cytometry (Fig. 1A). PcMab-47 recognized endogenous PODXL, which is expressed in LN229 (a glioblastoma cell line), whereas it did not react with LN229/PODXL-KO cells (PDIS-13) (Fig. 1B), indicating that PcMab-47 is specific against PODXL. The reaction of PcMab-47 against LN229/PODXL was higher than that against LN229 (Fig. 1B).

PODXL is expressed in many cancers, such as breast cancers $^{(22-24)}$ and colorectal cancers. ${ }^{(26-29)}$ We performed flow cytometry using several types of cancer cells and normal cells. PcMab-47 reacted with the cancer cell lines, such as Caco-2 (colon adenocarcinoma) and MDA-MB-468 (breast cancer) (Fig. 1C). PODXL is also expressed in normal tissues of kidney and ECs. ${ }^{(4)}$ PcMab-47 reacted with the normal cell lines, including HEK-293T (renal epithelial cell) and with vascular VECs (Fig. 1C). These results show the usefulness of PcMab-47 for detecting PODXL in flow cytometry.

We next investigated whether the epitope of PcMab-47 includes $\mathrm{N}$-glycan or $O$-glycan because PODXL is highly glycosylated (Fig. 1D, E). As shown in Figure 1D, PcMab-47 reacted with all glycan-deficient PODXL-expressing $\mathrm{CHO}$ cells, specifically Lec1/PODXL ( $N$-glycan-deficient), Lec2/ PODXL (sialic acid-deficient), Lec8/PODXL (galactosedeficient), and Lec13/PODXL (fucose-deficient). Furthermore, PcMab-47 reacted with all glycan-deficient HEK-293T cells such as PDIS-12 ( $N$-glycan-deficient), PDIS-22 (sialic acid-deficient), PDIS-18 (galactose-deficient), and PDIS-20 ( $N$-glycan/sialic acid/galactose-deficient), indicating that glycans are not included in the PcMab-47 epitope (Fig. 1E).

Next, we investigated the immunohistochemical utility of PcMab-47 in normal tissues or cancers. As shown in Figure 2, PcMab-47 stained podocytes or ECs of kidney (Fig. 2A, B). PcMab-47 reacted with colon adenocarcinoma, in which membrane/cytoplasmic-staining pattern was observed (Fig. 3A, B). Strong expression of PODXL was also observed in ECs in colon adenocarcinoma (Fig. 3B). In contrast, weak expression of PODXL was observed in cancer cells, but stronger expression was detected in ECs in breast cancer (Fig. 3C, D). These results indicate that PcMab-47 is very useful for immunohistochemical detection of both cancer cells and normal cells that express PODXL.

Therefore, PcMab-47 may facilitate investigation of the expression and function of PODXL in cancer and normal tissues. Future investigations should include production of different epitope-possessing anti-PODXL mAbs to elucidate the function of PODXL.

\section{Acknowledgments}

This work was supported, in part, by the Basic Science and Platform Technology Program for Innovative Biological Medicine from Japan Agency for Medical research and development, AMED (Y.K.), by the Translational Research Network Program from AMED (Y.K.), by the Platform for Drug Discovery, Informatics, and Structural Life Science (PDIS) from AMED (Y.K.), by project for utilizing glycans in the development of innovative drug discovery technologies from AMED (Y.K.), by the Regional Innovation Strategy Support Program from the Ministry of Education, Culture, Sports, Science and Technology (MEXT) of Japan (Y.K.), and by JSPS KAKENHI Grant Number 26440019 (M.K.K.) and 16K10748 (Y.K.). This work was performed, in part, under the Cooperative Research Program of Institute for Protein Research, Osaka University, CR-16-05, and by the Grant for Joint Research Project of the Institute of Medical Science, the University of Tokyo. The authors would like to thank Takeshi Murata (Chiba University), Hiroaki Uchida (The University of Tokyo), and Hideaki Tahara (The University of Tokyo) for their specialized advice, and also thank Enago (www.enago.jp) for the English language review.

\section{Author Disclosure Statement}

No competing financial interests exist.

\section{References}

1. McNagny KM, Pettersson I, Rossi F, Flamme I, Shevchenko A, Mann M, and Graf T: Thrombomucin, a novel cell surface protein that defines thrombocytes and multipotent hematopoietic progenitors. J Cell Biol 1997;138:1395-1407.

2. Schopperle WM, Kershaw DB, and DeWolf WC: Human embryonal carcinoma tumor antigen, Gp200/GCTM-2, is 
podocalyxin. Biochem Biophys Res Commun 2003;300: 285-290.

3. Schopperle WM, and DeWolf WC: The TRA-1-60 and TRA-1-81 human pluripotent stem cell markers are expressed on podocalyxin in embryonal carcinoma. Stem Cells 2007;25:723-730.

4. Doyonnas R, Kershaw DB, Duhme C, Merkens H, Chelliah S, Graf T, and McNagny KM: Anuria, omphalocele, and perinatal lethality in mice lacking the CD34-related protein podocalyxin. J Exp Med 2001;194:13-27.

5. Berenson RJ, Andrews RG, Bensinger WI, Kalamasz D, Knitter G, Buckner CD, and Bernstein ID: Antigen CD34+ marrow cells engraft lethally irradiated baboons. J Clin Invest 1988;81:951-955.

6. Hayatsu N, Kaneko MK, Mishima K, Nishikawa R, Matsutani M, Price JE, and Kato Y: Podocalyxin expression in malignant astrocytic tumors. Biochem Biophys Res Commun 2008;374:394-398.

7. Kerjaschki D, Sharkey DJ, and Farquhar MG: Identification and characterization of podocalyxin - the major sialoprotein of the renal glomerular epithelial cell. J Cell Biol 1984;98:1591-1596.

8. Kerjaschki D, Poczewski H, Dekan G, Horvat R, Balzar E, Kraft N, and Atkins RC: Identification of a major sialoprotein in the glycocalyx of human visceral glomerular epithelial cells. J Clin Invest 1986;78:1142-1149.

9. Kershaw DB, Beck SG, Wharram BL, Wiggins JE, Goyal M, Thomas PE, and Wiggins RC: Molecular cloning and characterization of human podocalyxin-like protein. Orthologous relationship to rabbit PCLP1 and rat podocalyxin. J Biol Chem 1997;272:15708-15714.

10. Larrucea S, Butta N, Rodriguez RB, Alonso-Martin S, Arias-Salgado EG, Ayuso MS, and Parrilla R: Podocalyxin enhances the adherence of cells to platelets. Cell Mol Life Sci 2007;64:2965-2974.

11. Larrucea S, Butta N, Arias-Salgado EG, Alonso-Martin S, Ayuso MS, and Parrilla R: Expression of podocalyxin enhances the adherence, migration, and intercellular communication of cells. Exp Cell Res 2008;314:20042015.

12. Takeda T, McQuistan T, Orlando RA, and Farquhar MG: Loss of glomerular foot processes is associated with uncoupling of podocalyxin from the actin cytoskeleton. J Clin Invest 2001;108:289-301.

13. Takeda T: Podocyte cytoskeleton is connected to the integral membrane protein podocalyxin through $\mathrm{Na}+\mathrm{H}+-$ exchanger regulatory factor 2 and ezrin. Clin Exp Nephrol 2003;7:260-269.

14. Tan PC, Furness SG, Merkens H, Lin S, McCoy ML, Roskelley CD, Kast J, and McNagny KM: Na+/H+ exchanger regulatory factor-1 is a hematopoietic ligand for a subset of the CD34 family of stem cell surface proteins. Stem Cells 2006;24:1150-1161.

15. Tateno H, Matsushima A, Hiemori K, Onuma Y, Ito Y, Hasehira K, Nishimura K, Ohtaka M, Takayasu S, Nakanishi M, Ikehara Y, Nakanishi M, Ohnuma K, Chan T, Toyoda M, Akutsu H, Umezawa A, Asashima M, and Hirabayashi J: Podocalyxin is a glycoprotein ligand of the human pluripotent stem cell-specific probe rBC2LCN. Stem Cells Transl Med 2013;2:265-273.

16. Binder ZA, Siu IM, Eberhart CG, Ap Rhys C, Bai RY, Staedtke V, Zhang H, Smoll NR, Piantadosi S, Piccirillo SG, Dimeco F, Weingart JD, Vescovi A, Olivi A, Riggins GJ, and Gallia GL: Podocalyxin-like protein is expressed in glioblastoma multiforme stem-like cells and is associated with poor outcome. PLoS One 2013;8:e75945.

17. Casey G, Neville PJ, Liu X, Plummer SJ, Cicek MS, Krumroy LM, Curran AP, McGreevy MR, Catalona WJ, Klein EA, and Witte JS: Podocalyxin variants and risk of prostate cancer and tumor aggressiveness. Hum Mol Genet 2006;15:735-741.

18. Hsu YH, Lin WL, Hou YT, Pu YS, Shun CT, Chen CL, Wu YY, Chen JY, Chen TH, and Jou TS: Podocalyxin EBP50 ezrin molecular complex enhances the metastatic potential of renal cell carcinoma through recruiting Rac1 guanine nucleotide exchange factor ARHGEF7. Am J Pathol 2010;176:3050-3061.

19. Lin CW, Sun MS, and Wu HC: Podocalyxin-like 1 is associated with tumor aggressiveness and metastatic gene expression in human oral squamous cell carcinoma. Int $\mathbf{J}$ Oncol 2014;45:710-718.

20. Yasuoka H, Tsujimoto M, Hirokawa M, Tori M, Nakahara M, Miyauchi A, Kodama R, Sanke T, and Nakamura Y: Podocalyxin expression in undifferentiated thyroid carcinomas. J Clin Pathol 2008;61:1228-1229.

21. Boman K, Larsson AH, Segersten U, Kuteeva E, Johannesson H, Nodin B, Eberhard J, Uhlen M, Malmstrom PU, and Jirstrom K: Membranous expression of podocalyxinlike protein is an independent factor of poor prognosis in urothelial bladder cancer. Br J Cancer 2013;108:2321-2328.

22. Somasiri A, Nielsen JS, Makretsov N, McCoy ML, Prentice L, Gilks CB, Chia SK, Gelmon KA, Kershaw DB, Huntsman DG, McNagny KM, and Roskelley CD: Overexpression of the anti-adhesin podocalyxin is an independent predictor of breast cancer progression. Cancer Res 2004;64:5068-5073.

23. Snyder KA, Hughes MR, Hedberg B, Brandon J, Hernaez DC, Bergqvist P, Cruz F, Po K, Graves ML, Turvey ME, Nielsen JS, Wilkins JA, McColl SR, Babcook JS, Roskelley $\mathrm{CD}$, and McNagny KM: Podocalyxin enhances breast tumor growth and metastasis and is a target for monoclonal antibody therapy. Breast Cancer Res 2015;17:46.

24. Graves ML, Cipollone JA, Austin P, Bell EM, Nielsen JS, Gilks CB, McNagny KM, and Roskelley CD: The cell surface mucin podocalyxin regulates collective breast tumor budding. Breast Cancer Res 2016;18:11.

25. Cipollone JA, Graves ML, Kobel M, Kalloger SE, Poon T, Gilks CB, McNagny KM, and Roskelley CD: The antiadhesive mucin podocalyxin may help initiate the transperitoneal metastasis of high grade serous ovarian carcinoma. Clin Exp Metastasis 2012;29:239-252.

26. Larsson A, Johansson ME, Wangefjord S, Gaber A, Nodin B, Kucharzewska P, Welinder C, Belting M, Eberhard J, Johnsson A, Uhlen M, and Jirstrom K: Overexpression of podocalyxin-like protein is an independent factor of poor prognosis in colorectal cancer. Br J Cancer 2011;105:666672.

27. Kaprio T, Fermer C, Hagstrom J, Mustonen H, Bockelman $\mathrm{C}$, Nilsson $\mathrm{O}$, and Haglund C: Podocalyxin is a marker of poor prognosis in colorectal cancer. BMC Cancer 2014; 14:493.

28. Kaprio T, Hagstrom J, Fermer C, Mustonen H, Bockelman C, Nilsson O, and Haglund C: A comparative study of two PODXL antibodies in 840 colorectal cancer patients. BMC Cancer 2014;14:494.

29. Larsson AH, Lehn S, Wangefjord S, Karnevi E, Kuteeva E, Sundstrom M, Nodin B, Uhlen M, Eberhard J, Birgisson H, and Jirstrom K: Significant association and synergistic adverse prognostic effect of podocalyxin-like protein and 
epidermal growth factor receptor expression in colorectal cancer. J Transl Med 2016;14:128.

30. Heby M, Elebro J, Nodin B, Jirstrom K, and Eberhard J: Prognostic and predictive significance of podocalyxin-like protein expression in pancreatic and periampullary adenocarcinoma. BMC Clin Pathol 2015;15:10.

31. Saukkonen K, Hagstrom J, Mustonen H, Juuti A, Nordling S, Fermer C, Nilsson O, Seppanen H, and Haglund C: Podocalyxin is a marker of poor prognosis in pancreatic ductal adenocarcinoma. PLoS One 2015;10:e0129012.

32. Laitinen A, Bockelman C, Hagstrom J, Kokkola A, Fermer $\mathrm{C}$, Nilsson $\mathrm{O}$, and Haglund C: Podocalyxin as a prognostic marker in gastric cancer. PLoS One 2015;10:e0145079.

33. Amo L, Tamayo-Orbegozo E, Maruri N, Eguizabal C, Zenarruzabeitia O, Rinon M, Arrieta A, Santos S, Monge J, Vesga MA, Borrego F, and Larrucea S: Involvement of platelet-tumor cell interaction in immune evasion. Potential role of podocalyxin-like protein 1. Front Oncol 2014;4:245.

34. Thomas SN, Schnaar RL, and Konstantopoulos K: Podocalyxinlike protein is an E-/L-selectin ligand on colon carcinoma cells: Comparative biochemical properties of selectin ligands in host and tumor cells. Am J Physiol Cell Physiol 2009;296:C505-C513.

35. Dallas MR, Chen SH, Streppel MM, Sharma S, Maitra A, and Konstantopoulos K: Sialofucosylated podocalyxin is a functional E- and L-selectin ligand expressed by metastatic pancreatic cancer cells. Am J Physiol Cell Physiol 2012; 303:C616-C624.

36. Laubli H, and Borsig L: Selectins promote tumor metastasis. Semin Cancer Biol 2010;20:169-177.

37. Oki H, Kaneko MK, Ogasawara S, Tsujimoto Y, Liu X, Sugawara M, Takakubo Y, Takagi M, and Kato Y: Characterization of monoclonal antibody LpMab-7 recognizing Non-PLAG domain of podoplanin. Monoclon Antib Immunodiagn Immunother 2015;34:174-180.

38. Oki H, Ogasawara S, Kaneko MK, Takagi M, Yamauchi M, and Kato Y: Characterization of monoclonal antibody LpMab-3 recognizing sialylated glycopeptide of podoplanin. Monoclon Antib Immunodiagn Immunother 2015;34: 44-50.
39. Kaneko MK, Oki H, Hozumi Y, Liu X, Ogasawara S, Takagi M, Goto K, and Kato Y: Monoclonal antibody LpMab-9 recognizes O-glycosylated N-terminus of human podoplanin. Monoclon Antib Immunodiagn Immunother 2015;34:310-317.

40. Kato Y, Ogasawara S, Oki H, Goichberg P, Honma R, Fujii Y, and Kaneko MK: LpMab-12 established by CasMab technology specifically detects sialylated O-Glycan on Thr52 of platelet aggregation-stimulating domain of human podoplanin. PLoS One 2016;11:e0152912.

41. Ogasawara S, Oki H, Kaneko MK, Hozumi Y, Liu X, Honma R, Fujii Y, Nakamura T, Goto K, Takagi M, and Kato Y: Development of monoclonal antibody LpMab-10 recognizing non-glycosylated PLAG1/2 domain including Thr34 of human podoplanin. Monoclon Antib Immunodiagn Immunother 2015;34:318-326.

42. Kato Y, Ogasawara S, Oki H, Honma R, Takagi M, Fujii Y, Nakamura T, Saidoh N, Kanno H, Umetsu M, Kamata S, Kubo H, Yamada M, Sawa Y, Morita K, Harada H, Suzuki H, and Kaneko MK: Novel monoclonal antibody LpMab17 developed by CasMab technology distinguishes human podoplanin from monkey podoplanin. Monoclon Antib Immunodiagn Immunother 2016;35:109-116.

Address correspondence to: Dr. Yukinari Kato New Industry Creation Hatchery Center Tohoku University 2-1 Seiryo-machi, Aoba-ku

Sendai Miyagi 980-8575 Japan

E-mail: yukinari-k@bea.hi-ho.ne.jp; yukinarikato@med.tohoku.ac.jp

Received: February 2, 2017 Accepted: March 9, 2017 